Louisiana State University

LSU Digital Commons

$1-1-2004$

\title{
Toxicity and behavioral effects of nootkatone, 1,10-dihydronootkatone, and tetrahydronootkatone to the formosan subterranean termite (Isoptera: Rhinotermitidae)
}

\author{
Sanaa A. Ibrahim \\ Minia University \\ Gregg Henderson \\ Louisiana State University \\ Betty C.R. Zhu \\ Louisiana State University \\ Huixin Fei \\ Louisiana State University \\ Roger A. Laine \\ Louisiana State University
}

Follow this and additional works at: https://digitalcommons.Isu.edu/biosci_pubs

\section{Recommended Citation}

Ibrahim, S., Henderson, G., Zhu, B., Fei, H., \& Laine, R. (2004). Toxicity and behavioral effects of nootkatone, 1,10-dihydronootkatone, and tetrahydronootkatone to the formosan subterranean termite (Isoptera: Rhinotermitidae). Journal of Economic Entomology, 97 (1), 102-111. https://doi.org/10.1093/ jee/97.1.102

This Article is brought to you for free and open access by the Department of Biological Sciences at LSU Digital Commons. It has been accepted for inclusion in Faculty Publications by an authorized administrator of LSU Digital Commons. For more information, please contact ir@lsu.edu. 


\title{
Toxicity and Behavioral Effects of Nootkatone, 1,10-Dihydronootkatone, and Tetrahydronootkatone to the Formosan Subterranean Termite (Isoptera: Rhinotermitidae)
}

\author{
SANAA A. IBRAHIM, ${ }^{1,2}$ GREGG HENDERSON, ${ }^{2,3}$ BETTY C. R. ZHU, ${ }^{2,4}$ HUIXIN FEI, ${ }^{2}$ \\ AND ROGER A. LAINE ${ }^{2,4}$
}

\section{J. Econ. Entomol. 97(1): 102-111 (2004)}

\begin{abstract}
Toxicity and behavioral effects of nootkatone and two of its derivatives, 1,10-dihydronootkatone and tetrahydronootkatone, to Coptotermes formosanus Shiraki were investigated on workers from two different colonies by using topical application assays, repellency assays, and sand barrier assays. The acute toxicity of the nootkatones on workers from both colonies increased as the saturation of the molecule increased, but the difference was significant for only one colony. The results of the repellency assays showed a similar trend of efficiency; the threshold concentration for significant repellency was four-fold higher in nootkatone treatments $(50 \mathrm{ppm})$ than in the reduced derivatives 1,10-dihydronootkatone or tetrahydronootkatone $(12.5 \mathrm{ppm})$. In sand barrier assays, a concentration of $100 \mathrm{ppm}$ of any of the three chemicals significantly reduced termite survival, tunnel building, and food consumption after a 12-d exposure. Termites preexposed to $100 \mathrm{ppm}$ nootkatone-treated sand and placed in containers without nootkatone for $15 \mathrm{~d}$ continued to exhibit abnormal feeding and digging behaviors; survivorship, tunneling, and feeding activities were significantly reduced by 83.5, 63.2, and $95.4 \%$, respectively. Termites pretreated for $12 \mathrm{~d}$ at concentrations of 50 and $75 \mathrm{ppm}$ nootkatone and tetrahydronootkatone returned to normal digging activity after they were removed from the treatments, but their feeding activity was significantly reduced.
\end{abstract}

KEY WORDS Coptotermes formosanus, nootkatone, 1,10-dihydronootkatone, tetrahydronootkatone

The Formosan SUBTERRANEAN TERMITE, Coptotermes formosanus Shiraki, is considered one of the most destructive insect pests in the United States ( $\mathrm{Su}$ and Tamashiro 1987). It can destroy buildings after 2 yr of infestation and cause serious harm to shade trees (Spink 1967, Tamashiro et al. 1987, Henderson 2001). In Louisiana, the Formosan subterranean termite was first identified in Lake Charles and New Orleans in 1966 (Spink 1967). It has spread across the south, infesting up to $30 \%$ of the trees in certain locations of southern Louisiana (Henderson 2001). Of the estimated $\$ 138$ billion in damage that all invasive species cause in the United States every year, Formosan subterranean termites contribute $\$ 1.5$ billion (http:// mirir.tulane.edu/ispecies/Irq1.html). The cost to control and repair the damage by Formosan subterranean termites in Louisiana alone was estimated at $\$ 500$ million to $\$ 1$ billion; control and repair costs are estimated at $\$ 300$ million annually in New Orleans alone (Suszkiw 1998).

\footnotetext{
${ }^{1}$ Department of Plant Protection, Faculty of Agriculture, Minia University, Minia, Egypt.

${ }^{2}$ Department of Entomology, Louisiana State University Agricultural Center, Baton Rouge, LA 70803.

${ }^{3}$ Corresponding author. E-mail: grhenderson@agctr.lsu.edu.

${ }^{4}$ Department of Biological Sciences, Louisiana State University, Baton Rouge, LA 70803.
}

Over the past $50 \mathrm{yr}$, synthetic insecticides from several chemical groups have been used to control the Formosan subterranean termite. Many were reported to cause serious problems. For example, cyclodienes were effectively used to control termites; however they are no longer used because of their risk to the environment and human health (Sim et al. 1998). Likewise, in addition to human health concerns, some organophosphate insecticides have been reported to cause air pollution (Katsura et al. 1996) and contamination of small ponds (Carr et al. 1997, Kumar and Chapman 2001). Most recently, one of the newest registered termiticides, fipronil, has been reported to induce thyroid cancer (http://www.fluoridealert. org/pesticides/Fipronil.article.Pest.News.htm). Variation in Formosan subterranean termite susceptibility to termiticides is being reported in several states ( $\mathrm{Su}$ et al. 1997, Osbrink et al. 2001).

Searching for safe botanical insecticides is an approach currently under investigation for insect management (EPA 1996, Kumar and Chapman 2001, Kumari et al. 2002). Essential oils, widely used as fragrances and flavors in the perfume and food industries, have long been reputed to repel insects and have been evaluated for their use in controlling termites (Supriana 1983, Grace and Yates 1992, Wilkins 1992, Cornelius et al. 1997). Vetiver oil, an extract of the 
roots of Vetiveria zizanioides Linn Nash, used in the perfume industry, is repellent to insects (Irvine 1930 stated in Jain et al. 1982) and has potent topical irritant activity on cockroaches and flies (Jain et al. 1982). The activity of vetiver oil seems to be largely restricted to the sesquiterpene ketones it contains (Jain et al. 1982). Vetiver oil is a repellent and toxicant to the Formosan subterranean termite (Maistrello et al. 2001a, Zhu et al. 2001a).

Nootkatone, an eudalenoid sesquiterpene ketone, was originally isolated from the heartwood of Alaskan yellow cedar, Chamaecyparis nootkatensis (D. Don) (Erdtman and Hirose 1962). It is also a major constituent of grapefruit oil (Macleod and Buigues 1964) and an isomer of an insect-active sesquiterpene ketone from vetiver oil, $\alpha$-vetivone, (Jain et al. 1982). Like vetiver oil, nootkatone is also used in the perfume and flavor industries (Shaw and Wilson 1981). The first evaluation of nootkatone on C. formosanus as a termite repellent barrier was by Zhu et al. (2001b), and its repellency action was confirmed by Maistrello et al. (2001a,b, 2002). The objectives of this study were to quantify nootkatone toxicity on $C$. formosanus and to compare the toxicity and behavioral effects of nootkatone with two of its reduced derivatives.

\section{Materials and Methods}

Insects. Worker and soldier C. formosanus were collected from different locations in Louisiana by using a milk crate-trapping technique similar to that described by French (1994) with some modifications. Forty-four pine wood sticks ( 3.5 by 3.5 by $32 \mathrm{~cm}$ ) were placed in a plastic milk crate ( 33 by 33 by $26 \mathrm{~cm}$ ) in a grid formation. The crates were buried completely under ground near suspected termite-infested sites and left for 1 mo before inspection. Formosan subterranean termite-infested crates were brought back to the laboratory, held in 250-liter cans with the original source of food, and kept at room temperature (26$28^{\circ} \mathrm{C}$ ) and $70-80 \%$ RH. Each can was surrounded with a metal water trough to prevent termite escape and invasion by foraging ants. Termites were collected from New Orleans in December 1999 (colony L); Lake Charles on 28 June 2001 (colony C); New Orleans on 14 March 2002 (colony WB); Segnette State Park, New Orleans on 24 April 2002 (colony B); and an island on Calcasieu River, Lake Charles, on 15 January 2003 (colony CR). When termites were needed, they were gently knocked from the wood sticks into clean plastic trays $(40$ by $50 \mathrm{~cm}$ ) and separated from debris by allowing them to cling to sheets of moistened paper towels.

Chemicals. Technical grade nootkatone, crystalline $97.0 \%$ (AI) [4,4a, 5,6,7,8-hexahydro-6-isopropenyl4,4a-dimethyl-2 $\left({ }^{1} H\right)$-naphthalenone] was purchased from Lancaster Synthesis Inc. (Windham, NH). 1,10Dihydronootkatone $85 \%$ (AI) $[1,4,4 a, 5,6,7,8,10$ octahydro-6-isopropenyl-4,4a-dimethyl- $2\left({ }^{1} H\right)$ naphthalenone] and tetrahydronootkatone $85 \%$ (AI) $[1,4,4 a, 5,6,7,8,10,11,12$-octahydro-6-isopropyl-4,4adimethyl-2 $\left({ }^{1} H\right)$-naphthalenone] were obtained from

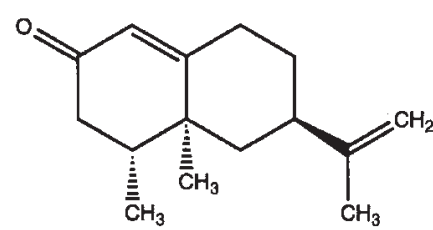

Nootkatone

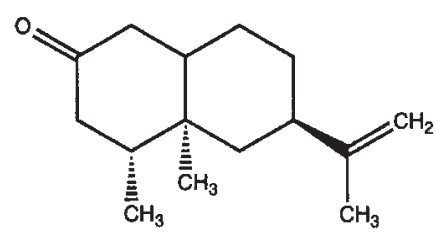

1,10-dihydronootkatone

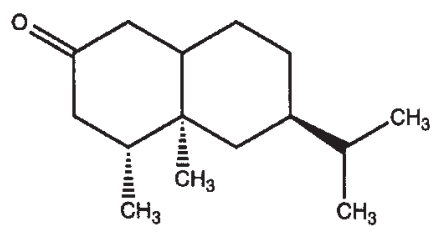

Tetrahydronootkatone

Fig. 1. Chemical structures of nootkatone, 1,10-dihydronootkatone, and tetrahydronootkatone.

Subcon Products, Inc. (Totowa, NJ). The chemical structures of the three chemicals are shown in Fig. 1.

Purification of 1,10-Dihydronootkatone and Tetrahydronootkatone. One gram of each chemical was dissolved in $5 \mathrm{ml}$ of $95 \%$ ethyl alcohol mixed with $5 \mathrm{~g}$ of silica gel and air-dried for $15 \mathrm{~min}$. A glass column (2 by $30 \mathrm{~cm}$ ) was used for purification. The silica gel containing the chemical sample was applied to the bottom of the column and then completely filled with silica gel. Chloroform was used to develop the sample (ascending run). The silica gel was forced out from the column after the chloroform reached the top. Every $2.5 \mathrm{~cm}$, a section of silica gel was collected in a flask and extracted with $40 \mathrm{ml}$ of ethyl alcohol. The fractions were tested on thin layer chromatography and the purified chemicals ( $>97 \%$ purity) were analyzed using gas chromatography-mass spectroscopy.

Topical Application Bioassays. Acute toxicity and delayed effects of nootkatone and its derivatives were determined on workers from colony $\mathrm{C}$ and colony $\mathrm{L}$.

Acute Toxicity. Seven doses ranging from 6.25 to $200 \mu \mathrm{g} /$ insect were used to establish the probit analysis results of each chemical. For each tested colony, five replicates of 50 workers were used. Termites were placed in petri dishes $(60$ by $15 \mathrm{~mm}$ ) and chilled on top of an ice brick ( 30.32 by 17.78 by $3.81 \mathrm{~cm}$ ) (IceBerg, Mid-Lands Chemical Company Inc., Miami, FL) to slow their movement (Sun et al. 2003). Each dose was topically applied in $0.2 \mu \mathrm{l}$ of ethanol to the dorsum of the mesothorax by using a Hamilton PB 600 repeating dispenser equipped with a $10-\mu$ l syringe (Hamilton, 
Reno, NV). Control workers were treated with $0.2 \mu \mathrm{l}$ of ethanol only. After treatment, termites were held in plastic containers ( 55 by $37 \mathrm{~mm}$ ), supplied with Whatman no. 2 filter paper ( $55 \mathrm{~mm}$ in diameter) moistened with $250 \mu$ l of double distilled water $\left(\mathrm{DDH}_{2} \mathrm{O}\right)$. Five untreated soldiers were added to each replicate to maintain the normal proportion of soldiers in the termite population (Haverty 1977). Treated termites were incubated for $3 \mathrm{~d}$ at $27.4^{\circ} \mathrm{C}$ and $70 \% \mathrm{RH}$, and daily mortality of workers was assessed. Dose-mortality data were analyzed by probit analysis as adapted for PC use (POLO PC), which automatically corrected for control mortality using Abbott's transformation (LeOra Software 1987).

Delayed Effects. Workers that were topically treated for evaluation of the $72-\mathrm{h}_{25}$ values of each chemical were incubated $14 \mathrm{~d}$ to study the delayed effects on termite survivorship and feeding activity. Workers from colonies C and L were topically treated using the same technique as described above; however, the plastic containers used to hold the termites were provided with $10 \mathrm{~g}$ of moistened sand (Blasting fine sand no. 4, Cement Products Inc., Baton Rouge, LA) in addition to the weighed filter paper. Sand was autoclaved for $30 \mathrm{~min}$, oven-dried, and then mixed with $1 \mathrm{ml}$ of $\mathrm{DDH}_{2} \mathrm{O}$ before loading in the container. On day 14, the number of living workers was counted, and filter paper from each replicate was cleaned and dried at $60^{\circ} \mathrm{C}$ for $24 \mathrm{~h}$ and reweighed to determine food consumption. To evaluate the effect of treatments on termite survival and food consumption, an analysis of variance (ANOVA) (SAS GLM Procedure) was conducted followed by Tukey's Studentized range (honestly significant difference) test (SAS Institute 1989). All data were judged at $\alpha=0.05$.

Repellency Assays. This study was conducted as described by Smith (1979) with some modification. Agar was prepared by heating and stirring $1.0 \mathrm{~g}$ in 100 $\mathrm{ml}$ of $\mathrm{DDH}_{2} \mathrm{O}$ and then $1 \mathrm{ml}$ of warm agar solution was spread evenly on the bottom of a plastic petri dish (60 by $15 \mathrm{~mm}$ ). For each chemical and colony, concentrations in $0.2 \mathrm{ml}$ of ethyl alcohol aliquots were mixed with $1 \mathrm{~g}$ of autoclaved sand. Five replicates of each treatment and ethanol-treated sand alone (control) were prepared. Treated sand was kept for $2 \mathrm{~h}$ in the fume hood at room temperature for ethanol evaporation before adding it to one side of the agar layer. The other side was covered with $1 \mathrm{~g}$ of untreated sand. Ten workers were placed along the borderline between the treated and untreated sand. The five replicates of each treatment were covered with the lids and aluminum foil was placed over top to reduce the light. Counts of termites in the treated side were recorded every $15 \mathrm{~min}$ in the first hour, and every $30 \mathrm{~min}$ during the next $3 \mathrm{~h}$, after which counts were recorded twice at 1 -h intervals with a final count at $24 \mathrm{~h}$. For each colony, the mean percentages of termite workers in the treated side were subjected to ANOVA as described previously.

Sand Barrier Assays. Two experiments were conducted to study the performance of nootkatone and its derivatives on termite survival, feeding, and tunneling activities by using termite from two colonies (WB and CR).

Experiment 1. Three-chambered transparent plastic containers ( 18.0 by 8.0 by $4.0 \mathrm{~cm}$ each box, 5.6 by 8.0 by $4.0 \mathrm{~cm}$ each chamber Pioneer Packaging Co., North Dixon, KY) were used. Small holes $(0.5 \mathrm{~cm}$ in diameter) were made at the bottom of each of the two inner walls connecting the three chambers to give termites the opportunity to cross the sand barrier and reach the food source. Concentrations of nootkatone and tetrahydronootkatone were prepared by dissolving $18.75,28.13$, and $37.50 \mathrm{mg}$ (AI) in $50 \mathrm{ml}$ absolute ethanol. Then, $10 \mathrm{ml}$ of each solution was mixed with $75 \mathrm{~g}$ of autoclaved sand in plastic containers ( 115 by $37 \mathrm{~mm}$ ). For nootkatone and tetrahydronootkatone, the final concentrations in the sand were 50, 75, and $100 \mathrm{ppm}$ (wt:wt). Because of the small amount of 1,10-dihydronootkatone we had available, only 100 ppm (wt:wt) was tested. Five replicates were assayed for each concentration. Sand in the control treatments was treated with ethanol only. The containers having untreated and treated sand were kept overnight uncovered in a fume hood at room temperature. After being dried, $7.5 \mathrm{ml}$ of $\mathrm{DDH}_{2} \mathrm{O}$ was mixed with the sand and added into the middle chamber. The moistened sand was packed and leveled ( $2 \mathrm{~cm}$ in thickness) to allow clear visualization of tunnels. Two filter papers (Whatman no. 2) were weighed and placed in one side of the two lateral chambers and then wetted with 500 $\mu \mathrm{l}$ of $\mathrm{DDH}_{2} \mathrm{O}$. A group of 150 workers and 15 soldiers (colony WB) was released in the opposite side chamber. Termites were required to cross a sand barrier in the middle chamber via tunneling or by walking on the surface of the treated sand to reach the feeding chamber. Containers were covered and incubated in darkness at $27.4^{\circ} \mathrm{C}$ and $70 \% \mathrm{RH}$. On days 1,3 , and 6 , the number of workers was recorded in the releasing and feeding chambers. Twelve days after treatment the bottoms of the containers were scanned (ScanJet 4C, Hewlett Packard, Palo Alto, CA) to fix the image of the tunnels and were printed actual size to measure tunnel lengths (using a string and ruler). Also at that time, living workers were counted and filter papers were cleaned, dried, and weighed as described previously.

To evaluate the effects of treatments on termite survival, tunnel building, and food consumption, data were subjected to ANOVA as described previously. Also, paired $t$-tests were performed to compare the percentage of termites in releasing and feeding chamber of each treatment (SAS Institute 1989).

Experiment 2. This experiment was conducted to confirm the results obtained in experiment 1 with colony WB. Termites from colony CR were used and the three chemicals were applied to sand at $100 \mathrm{ppm}$. A starvation treatment was included exactly the same as the control treatment except that no food was provided. This treatment was added to answer the question of whether mortality was a direct function of starvation. Mortality was recorded every $3 \mathrm{~d}$ for $12 \mathrm{~d}$. Tunnel lengths and food consumption were measured on day 12 as described previously. Among treatments, percentages of mortality, tunnel lengths, and filter 
paper consumption were subjected to ANOVA as described previously.

Latent Effects. This experiment was conducted to study the effects of the three chemicals after termites were removed from the treatments for $15 \mathrm{~d}$. Termites from colony WB previously exposed for $12 \mathrm{~d}$ to different concentrations of nootkatone $(50,75$, and 100 ppm) and tetrahydronootkatone (50 and $75 \mathrm{ppm}$ ) in sand barriers (experiment 1) were removed from the treatments and the latent effects of chemical treatments on termite survival, tunneling, and feeding activities were recorded after $15 \mathrm{~d}$. After experiment 1 was ended on day 12 , living termites from all replicates of each treatment were collected and used for this experiment. The previously described techniques and conditions were used except that the middle chamber contained only untreated sand. One hundred workers were released in one of the two lateral side chamber and two filter papers were placed in the opposite side chamber. The number of replicates varied from five in the control treatment to two in the 75 ppm tetrahydronootkatone treatment (based on the number of termites collected from experiment 1 ). After $15 \mathrm{~d}$, the number of living workers was counted. Tunnel lengths and filter paper consumption were measured as described previously. Among treatments, mean percentages of survival, tunnel lengths, and filter paper consumption were subjected to ANOVA as described previously.

\section{Results}

Topical Application Bioassays. Acute Toxicity. Tetrahydronootkatone was significantly more toxic on workers from colony $\mathrm{C}$ than nootkatone (based on the nonoverlap of the $95 \%$ confidence limits (CL) of the $24 \mathrm{~h} \mathrm{LD}_{50}$ values; Table 1). Toxicity of 1,10-dihydronootkatone on workers from both colonies was not significantly different from that of nootkatone or tetrahydronootkatone. Probit analysis results established from the 72-h mortality data indicated that chi-square values were not significant (except for tetrahydronootkatone with colony C; Table 1), indicating a good fit of mortality data on the probit lines. The toxicity of the three chemicals on workers from the two colonies was not significantly different (based on the overlap of the $95 \%$ CL of the 72-h $\mathrm{LD}_{50}$ values and the $72-\mathrm{h} \mathrm{LD}_{90}$ values). The intercolony variation in the toxicity response to the three chemicals also was not significant. All dead workers that had been treated at $\geq 50$ micrograms per insect had black spots beneath the cuticle, in the proximity to the epidermal cells in the head and leg areas.

Delayed Effects. Survivorship of workers was significantly reduced in the chemical treatments compared with the control (Fig. 2). Survivorship of termites in nootkatone treatments was significantly higher than that for tetrahydronootkatone (colonies $\mathrm{C}$ and L) or 1,10-dihydronootkatone (colony C only). The average food consumption was significantly reduced in treatments of 1,10-dihydronootkatone and

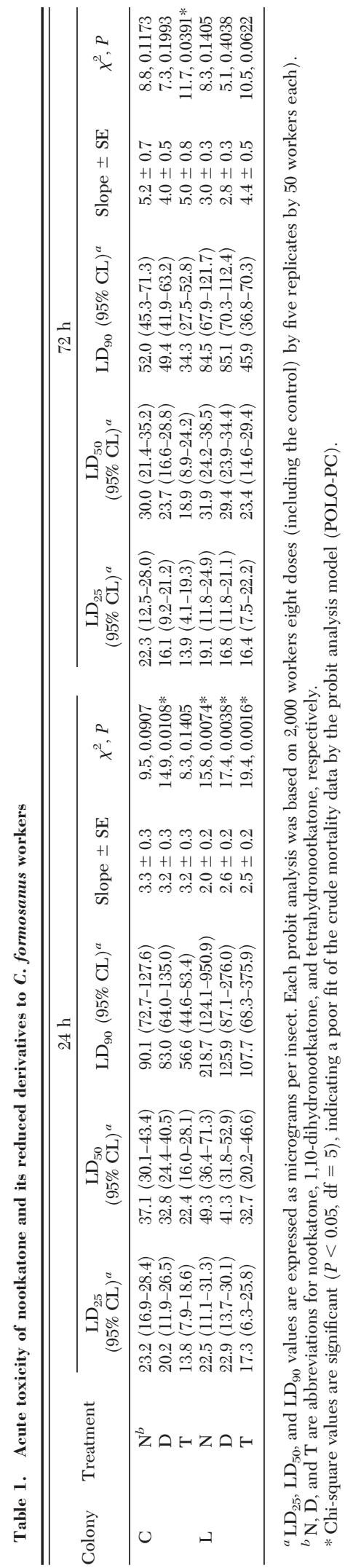

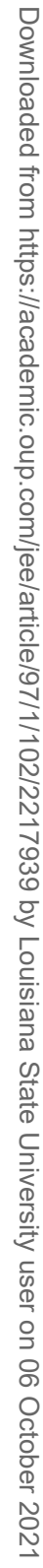




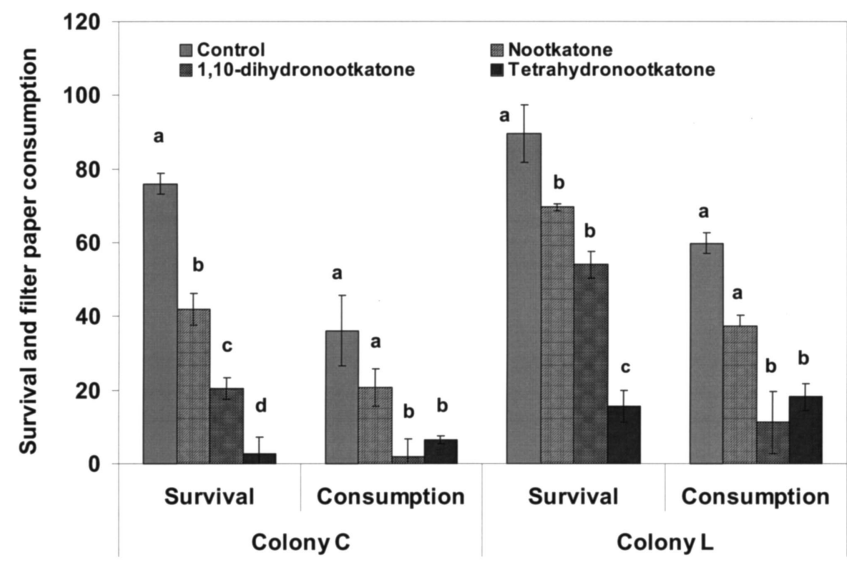

Fig. 2. Means ( $\pm \mathrm{SE}$ ) of C. formosanus survival (percentage) and food consumption (milligrams) $14 \mathrm{~d}$ after C. formosanus workers were topically treated with the $72-\mathrm{h} \mathrm{LD}_{25}$ of nootkatone and two of its derivatives. For each group, among treatments, means followed by the same letter are not significantly different $(P>0.05)$. $F$ and $P$ values are 20.95, <0.0001; 9.21, 0.0009; 21.57, <0.0001; and 13.09, <0.0001, respectively, for survival, colony C; food consumption, colony C; survival, colony L; and food consumption colony $\mathrm{L}$. The df values are 3,16 for all.

tetrahydronootkatone, but not for nootkatone (Fig. 2).

Repellency Assays. Nootkatone at levels of 12.5 and $25.0 \mathrm{ppm}$ (micrograms per gram of sand) was not an effective repellent (Table 2). However, the percentage of termites (from both colonies) found in sand treated with 1,10-dihydronootkatone and tetrahydronootkatone at $\geq 12.5 \mathrm{ppm}$ was significantly less than in the controls. Nootkatone repelled termites at $50 \mathrm{ppm}$ but was less repellent than its derivatives at this level (colonies C and B). At 100 ppm, there were no significant differences among the three chemicals (Table 2).

Sand Barrier Assays. Experiment 1. The mean percentage of termites in the releasing chambers was significantly higher for all chemical treatments compared with the control, indicating that termites were repelled from crossing the barrier (Table 3). On day 1 , the greatest number $(\geq 86 \%$ ) of individuals was found in the releasing chambers in 100 ppm tetrahydronootkatone and 100 ppm of 1,10-dihydronootkatone treatments compared with $13 \%$ in the control
(Table 3). The mean percentage of termites that crossed the chemical barriers into the feeding chambers was significantly less $(\leq 18 \%)$ compared with control (66\%). On day 3 , a higher percentage of workers stayed in the releasing chambers of the treatments $(36-87 \%)$ compared with $3 \%$ in the controls. The mean percentage of termites in the feeding chambers of controls was $59 \%$ compared with $0-28 \%$ in the feeding chambers of chemical treatments (Table 3 ). Termites found in the middle chamber of the chemical treatments behaved abnormally by remaining in clusters on the surface of treated sand. Those that made it to the feeding chamber did so not by tunneling in the treated sand, but by traveling along the surface of sand. This behavior was not observed in the controls; on day $6,99 \%$ of the termites in the control treatment were actively tunneling or feeding compared with $12-55 \%$ for the chemical treatments (Table 3 ).

Fewer and shorter tunnels were constructed in 75 and $100 \mathrm{ppm}$ tetrahydronootkatone, and $100 \mathrm{ppm}$ nootkatone-treated sand (Fig. 3). Tunnels constructed in 100 ppm 1,10-dihydronootkatone-treated

Table 2. Mean percentages $( \pm \mathrm{SE})$ of $C$. formosanus workers counted in ethanol- and chemically treated sand

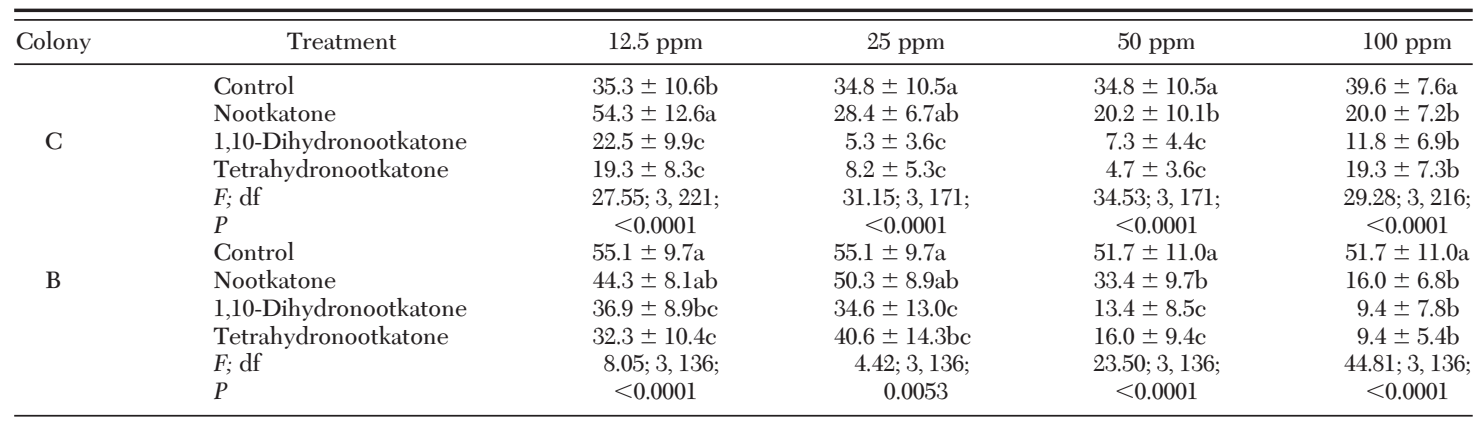

General averages of 13 readings during the first $24 \mathrm{~h}$ after treatment. The preliminary statistical analysis revealed no significant differences between the readings at different observation periods; therefore, they were considered as replications. 


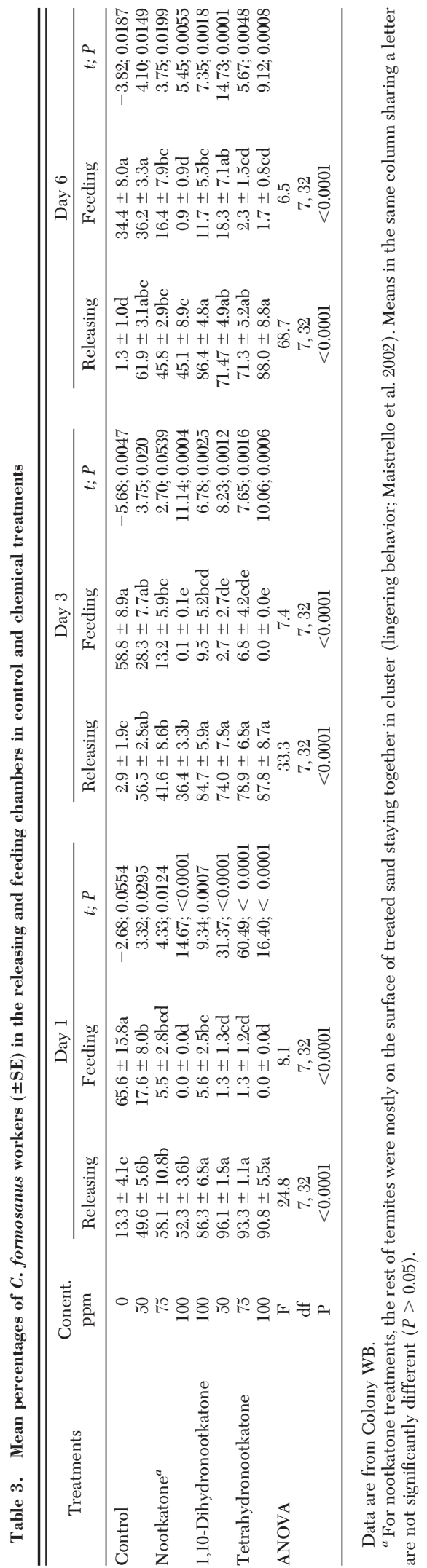

sand were significantly longer than those at $100 \mathrm{ppm}$ tetrahydronootkatone, but were not significantly different from those made in $100 \mathrm{ppm}$ nootkatone treatment.

At levels of $50 \mathrm{ppm}$ nootkatone and $50 \mathrm{ppm}$ tetrahydronootkatone, the average food consumption was not significantly different from the control (Fig. 3). The 100 ppm nootkatone, 100 ppm 1,10-dihydronootkatone, and 75-100 ppm tetrahydronootkatone treatments showed the greatest efficiency in reducing food consumption.

With the exception of $50 \mathrm{ppm}$ nootkatone, a significant reduction in termite survival was observed on day 12 (Fig. 3). Treatments of $100 \mathrm{ppm}$ nootkatone, $100 \mathrm{ppm}$ 1,10-dihydronootkatone, and $100 \mathrm{ppm}$ tetrahydronootkatone significantly reduced survivorship to 42,22 , and $1 \%$, respectively, compared with $73 \%$ in the controls.

Experiment 2. Nootkatone and its derivatives exhibited the same pattern of efficiency when they were assayed on workers from colony CR. Nootkatone was less effective as a repellent and a toxicant than its derivatives (Table 4 ). On day 6 , tetrahydronootkatone had the greatest mortality (98\%), followed by $1,10-$ dihydronootkatone $(78 \%)$ and nootkatone (50\%) compared with a significantly lower mortality in the controls (12\%). On day 12 , cumulative mortality was not significantly different among nootkatones. Tunneling and feeding were significantly reduced in the three treatments $(0.0-12.3 \mathrm{~cm}$ and $0.0-25.7 \mathrm{mg}) \mathrm{com}$ pared with the control $(34.1 \mathrm{~cm}$ and $126.6 \mathrm{mg})$. Tetrahydronootkatone completely inhibited tunneling and feeding activities.

Latent Effects. After 15 d, survivorship of termites pretreated with $100 \mathrm{ppm}$ nootkatone was significantly less than the controls (Fig. 4). Survivorship of termites pretreated with nootkatone and tetrahydronootkatone at 50 and $75 \mathrm{ppm}$ was not significantly different from the control. Termites pretreated with $100 \mathrm{ppm}$ nootkatone were not able to dig in the untreated sand as far $(18 \mathrm{~cm})$ as termites in the control $(49 \mathrm{~cm}$; Fig. 4 ). Food consumption by termites pretreated with either of the two chemicals was significantly reduced (Fig. 4).

\section{Discussion}

Nootkatone, 1,10-dihydronootkatone, and tetrahydronootkatone were effective repellents and toxicants on the Formosan subterranean termite. Incorporated into sand barriers, they markedly reduced tunneling and feeding behavior and caused an effect termed "lingering behavior," a sharply reduced activity profile in which termites collect together in clusters on the surface of treated sand (previously observed by Maistrello et al. 2001a).

The repellent effect of nootkatone and its derivatives may be a function of the olfactory and/or contact receptors located on the termite antennae. Olfactory neuroreceptor systems in insect antennae offer high sensitivity to specific molecular structures, resulting in neuronal motor responses (Pickett et al. 2001). Re- 


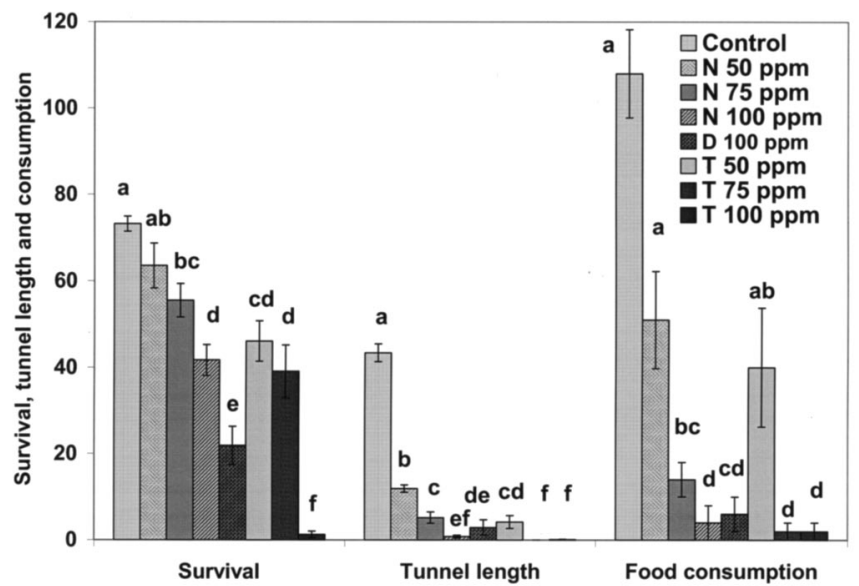

Fig. 3. Means $( \pm \mathrm{SE})$ of $C$. formosanus survival (percentage), tunnel length (centimeters), and food consumption (milligrams) in response to sand barriers treated with nootkatone and two of its derivatives. Colony WB was used and the data were recorded $12 \mathrm{~d}$ after treatment. For each group, among treatments, means followed by the same letter are not significantly different $(P>0.05)$. The $F$ values are 39.71, 30.48, and 12.70 for survival, tunnel length, and food consumption, respectively. $P$ and $\mathrm{df}$ values are $<0.0001$ and 7,32 for all.

cently, Ishida et al. (2002) isolated and identified a specific protein and two other cDNAs from the antennae of the dampwood termite, Zootermopsis nevadensis nevadensis (Hagen). GABA neuroreceptors (Eichenseer et al., 1998, González-Coloma et al., 2002) or taste receptors in the termite antennae (Kaib and Mikus 1999) may also be involved in avoidance behavior. Inhibition of sensory receptors of the mouthparts, resulting in disorientation of normal probing, feeding, and intake of food by insects can occur as a result of repellents (http://www.bicco.com/ bioneem.html).

Toxicity associated with repellent chemicals may in part be caused by starvation due to disruption of feeding behavior (Beck 1965). In our study, we controlled the time to $12 \mathrm{~d}$ to avoid such a starvation-mortality relationship. It has been shown that termites can live at least $10 \mathrm{~d}$ without food (Su and La Fage 1986), and we also confirmed in this study that starved termites lived at least $12 \mathrm{~d}$ without appreciable mortality when suitable moisture was provided.
In the topical application bioassays, nootkatone and its derivatives were toxic to Formosan subterranean termites when applied at relatively high doses compared with the recently registered termiticide fipronil (Ibrahim et al. 2003). However, compared with other natural products, they effectively repelled and killed C. formosanus at much lower concentrations $(\leq 100$ ppm) (Grace and Yates 1992, Cornelius et al. 1997).

There is no available literature pertaining to the mode of toxic action of nootkatone and its derivatives. Naturally occurring components in essential oils and vegetable oils may physically kill termites by covering the spiracles or by causing insect dehydration (Fenigstein et al. 2001), but interference with the nervous system is also possible (Isman et al. 1997). After topical applications of nootkatone or its reduced derivatives to the termites, shaking activity followed by lethargy was observed. It is possible that the nootkatones partially act as enzyme inhibitors or stimulators. Nootkatone inhibits CYP2A6 and CYP2C19 in human liver microsomes (Tassaneeyakul et al. 2000) and acetyl-

Table 4. Percentages of mortality, tunnel lengths, and food consumption when $C$. formosanus termites were exposed to 100 ppm-treated sand barriers with nootkatone and two reduced derivatives

\begin{tabular}{|c|c|c|c|c|c|c|}
\hline \multirow{2}{*}{ Treatment } & \multicolumn{4}{|c|}{ Percentages of cumulative mortality ${ }^{a}$} & \multirow{2}{*}{$\begin{array}{c}\begin{array}{c}\text { Tunnel } \\
\text { length }(\mathrm{cm})^{a}\end{array} \\
\text { Day } 12\end{array}$} & \multirow{2}{*}{$\frac{\begin{array}{c}\text { Consumption } \\
(\mathrm{mg})^{a}\end{array}}{\text { Day } 12}$} \\
\hline & Day 3 & Day 6 & Day 9 & Day 12 & & \\
\hline Control & $5.7 \pm 2.4$ & $9.9 \pm 3.2 \mathrm{c}$ & $11.9 \pm 3.9 \mathrm{c}$ & $20.5 \pm 3.3 b$ & $34.1 \pm 5.2 \mathrm{a}$ & $126.6 \pm 14.1 \mathrm{a}$ \\
\hline Starvation & $14.9 \pm 6.7$ & $17.1 \pm 7.5 \mathrm{c}$ & $18.0 \pm 7.8 \mathrm{c}$ & $21.9 \pm 6.0 \mathrm{~b}$ & $40.5 \pm 2.1 \mathrm{a}$ & \\
\hline Nootkatone & $17.9 \pm 7.1$ & $49.9 \pm 19.5 \mathrm{bc}$ & $67.2 \pm 16.3 b$ & $79.9 \pm 11.2 \mathrm{a}$ & $12.3 \pm 4.4 \mathrm{~b}$ & $25.7 \pm 7.2 \mathrm{~b}$ \\
\hline 1,10-Dihydronootkatone & $20.4 \pm 6.3$ & $78.1 \pm 16.3 \mathrm{ab}$ & $93.6 \pm 5.1 \mathrm{a}$ & $96.1 \pm 3.9 \mathrm{a}$ & $3.7 \pm 3.7 \mathrm{~b}$ & $4.1 \pm 4.1 b$ \\
\hline Tetrahydronootkatone & $24.8 \pm 6.6$ & $98.4 \pm 0.7 \mathrm{a}$ & $100.0 \pm 0.0 \mathrm{a}$ & $100.0 \pm 0.0 \mathrm{a}$ & $0.0 \pm 0.0 \mathrm{~b}$ & $0.0 \pm 0.0 \mathrm{~b}$ \\
\hline$F$ & 1.38 & 10.21 & 23.31 & 41.64 & 25.74 & 52.93 \\
\hline df & 4,20 & 4,20 & 4,20 & 4,20 & 4,20 & 6,16 \\
\hline$P$ & 0.2757 & 0.0001 & $<0.0001$ & $<0.0001$ & $<0.0001$ & $<0.0001$ \\
\hline
\end{tabular}

Data are from Colony CR.

${ }^{a}$ All data expressed as means \pm SE. Means in the same column sharing a letter are not significantly different $(P>0.05)$. 


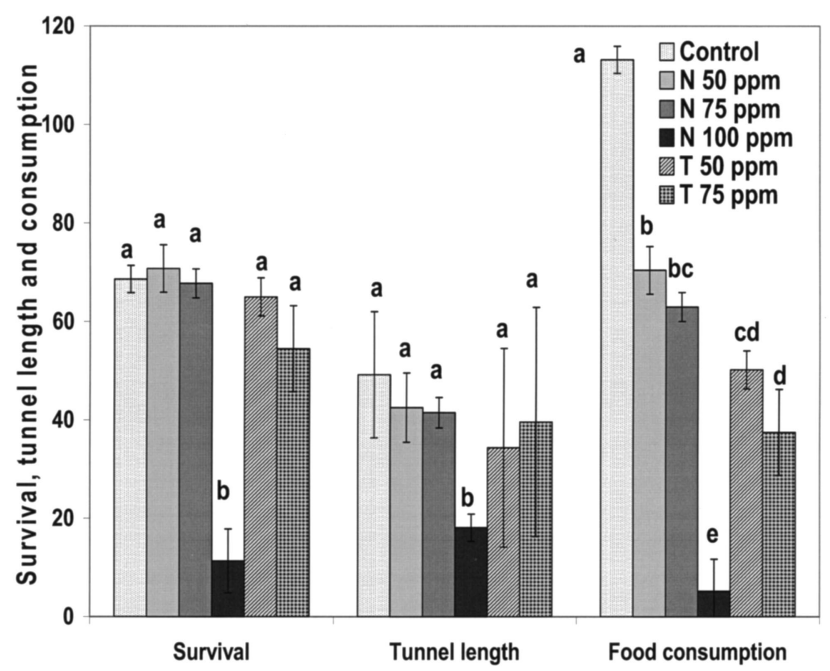

Fig. 4. Means ( $\pm \mathrm{SE}$ ) of C. formosanus survival (percentage), tunnel length (centimeters), and food consumption (milligrams) $15 \mathrm{~d}$ after pretreated C. formosanus termites were removed from the treatments. Termites were exposed in sand barrier assays to chemicals for $12 \mathrm{~d}$ and then living termites were transferred in equal numbers to untreated sand for $15 \mathrm{~d}$ to follow up the latent effects on termite survivorship, tunneling, and feeding activities. For each group, among treatments, means followed by the same letter are not significantly different $(P>0.05)$. $F$ and $P$ values are $17.85,<0.0001 ; 5.29,0.0054$; and 8.78, 0.0006, respectively, for survival, tunnel length, and food consumption. The df values are 5,15 for all.

cholinesterase from bovine erythrocytes (Miyazawa et al. 2001). An in vitro kinetic assay to measure acetylcholinesterase activity (from Formosan subterranean workers and soldiers; S.A.I. et al., unpublished data) revealed that nootkatone and tetrahydronootkatone at concentrations equivalent to the $24-\mathrm{h} \mathrm{LD}_{50}$ and $\mathrm{LD}_{90}$ significantly increased acetylcholinesterase activity. Such an effect could lead to a Myasthenia Gravis-like syndrome that occurs in humans (F. Enright, personal communication). This may explain the lethargic and lingering behavior of workers exposed to treated sand (Maistrello et al. 2001a, and data herein). Nootkatone and its derivatives may also affect muscle contraction and reduce the termite's ability to dig in treated sand. In addition, we observed that some of dead termites had black spots that occurred beneath the cuticle, in the proximity to the epidermal cells, in the head and leg areas. These spots are not fully characterized but could be caused by necrosis, pigment concentration, or a combination of the two. It seems that the dark pigment is being concentrated under the mandibles on the vertical section of the termite head, and pigmentation does not seem to be evenly distributed throughout the leg area. This may be a result of the chemicals entering the cuticle especially through the pore canals, dermal glands, and/or other cuticular structures, spreading and effectively preventing pigment produced by the epidermal cells to precipitate. The dark spots also could result from necrosis.

The variation in toxicity and repellency among nootkatone and its derivatives is probably related to the reductive substitutions that alter the three-dimensional structure, water solubility, alkalinity, vapor pressure, and volatility. The toxicity seems to be a function of the fused hydrocarbon rings and the ketone group. Saturating the double ring system by reducing the double bond (1,10-en) in the nootkatone molecule resulted in a $\geq 10 \%$ increase in the toxicity of 1,10-dihydronootkatone compared with nootkatone. Moreover, reducing the isopropenyl in 1,10-dihydronootkatone to isopropyl (in tetrahydronootkatone) resulted in an additional increase in toxicity $(\geq 20 \%)$.

It is interesting that certain plants such as grapefruit and Alaskan yellow cedar have developed potent antiinsect compounds such as the nootkatones, which must affect local ecological relationships, species, and numbers of insect pests around these plants. The chrysanthemum pyrethrins are also based on ring compounds with ketone groups, and it would be interesting to see whether their biological effects are based on similar receptor systems. Why more plants do not contain these potent compounds and why a large group of plants that are insect resistant have not developed from these examples is an interesting evolutionary question.

Previous publications with nootkatone (Zhu et al. 2001b, Maistrello et al. 2001a,b) and our current study with nootkatone and its reduced derivatives further confirm their efficiency against Formosan subterranean termites. Under laboratory conditions, nootkatone as a wood treatment maintained its initial activity for $1 \mathrm{yr}$ (Maistrello et al. 2002). Field evaluation of nootkatone and tetrahydronootkatone as wood treatments gave promising results (Nix et al. 2003). The relative safety of nootkatone for humans (Shaw and Wilson 1981) coupled with the above-mentioned results show promise of using these chemicals in termite control programs. 


\section{Acknowledgments}

We are indebted to Drs. S. Dean, R. Meola, and S. Meola for comments on the black spots associated with treated termites. We thank Y. Yu for assistance in the laboratory and R. K. Cross for literature searching. L. Mao, A. Sauer, and P. McLaughlin are thanked for reviewing this article. This project was partially supported by USDA-ARS New Orleans (58-6435-8-084) and approved for publication by the director of the Louisiana Agricultural Experiment Station as manuscript 02-14-0469.

\section{References Cited}

Beck, S. D. 1965. Resistance of plants to insects. Annu. Rev. Entomol. 10: 207-232.

Carr, R. L., L. L. Ho, and J. E. Chambers. 1997. Selective toxicity of chlorpyrifos to several species of fish during an environmental exposure: an investigation into the biochemical mechanisms behind this toxicity. Environ. Toxicol. Chem. 16: 2369-2374.

Cornelius, M. L., J. K. Grace, and J. R. Yates III. 1997. Toxicity of monoterpenoids and other natural products to the Formosan subterranean termite (Isoptera: Rhinotermitidae). J. Econ. Entomol. 90: 320-325.

Eichenseer, H., C. A. Mullin, and S. Chyb. 1998. Antifeedant discrimination thresholds for two populations of western corn rootworm. Physiol. Entomol. 23: 220-226.

[EPA] Environmental Protection Agency. 1996. Food Quality Protection Act. Public Law 104-170.

Erdtman, H., and Y. Hirose. 1962. The chemistry of the natural order Cupressales. 46. The structure of nootkatone. Acta Chem. Scand. 16: 1311-1314.

Fenigstein, A., M. Eliyahu, S. Gan-Mor, and D. Veierov. 2001. Effects of five vegetable oils on the sweetpotato whitefly Bemisia tabaci. Phytoparasitica 29: 197-206.

French, J. 1994. Termite bait box workshop: bait box technique for subterranean termite control. CSIRO, Division of Forest Products, Clayton, Victoria, Australia.

González-Coloma, A., F. Valencia, N. Martín, J. J. Hoffmann, L. Hutter, J. A. Marco, and M. Reina. 2002. Silphinene sesquiterpenes as model insect antifeedants. J. Chem. Ecol. 28: 117-129.

Grace, J. K., and J. R. Yates, 3rd. 1992. Behavioral effects of a neem insecticide on Coptotermes formosanus (Isoptera: Rhinotermitidae). Trop. Pest. Manage. 38: 176-180.

Haverty, M. I. 1977. The proportion of soldiers in termite colonies: a list and a bibliography (Isoptera). Sociobiology $2: 199-216$.

Henderson, G. 2001. Practical considerations of the Formosan subterranean termite in Louisiana: a 50-year-old problem. Sociobiology 37: 281-292.

Ibrahim, S. A., G. Henderson, and H. Fei. 2003. Toxicity, repellency, and horizontal transmission of fipronil in the Formosan subterranean termite (Isoptera: Rhinotermitidae). J. Econ. Entomol. 96: 461-467.

Irvine, F. R. 1930. Plants of the Gold Coast. Oxford University Press, London, England.

Ishida, Y., V. P. Chiang, M. I. Haverty, and W. S. Leal. 2002. Odorant-binding proteins from a primitive termite. J. Chem. Ecol. 28: 1887-1893.

Isman, M. B., L. B. Jeffs, C. A. Elliger, T. Miyake, and F. Matsumura. 1997. Petuniolides, natural insecticides from Petunia parodii, are antagonists of $\mathrm{GABA}_{\mathrm{a}}$ receptors. Pestic. Biochem. Physiol. 58: 103-107.

Jain, S. C., S. Nowicki, T. Eisner, and J. Meinwald. 1982 Insect repellents from vetiver oil: zizanal and epizizanal. Tetrahedron Lett. 23: 4639-4642.
Kaib, M., and S. Mikus. 1999. Neurophysiology of a taste receptor cell is a termite: evidence for two distinct receptor sites-water and sodium. In The 16th Annual Meeting of the International Society of Chemical Ecology (ISCE), 13-17 November 1999, Concorde Palm-Beach Hôtel, Marseille, France.

Katsura, E., H. Ogawa, H. Kojima, and A. Fukushima. 1996. Indoor air pollution by chlorpyrifos and S-421 after application for termite control. Jpn. J. Toxicol. Environ. Health. 42: 354-359.

Kumar, A., and J. C. Chapman. 2001. Profenofos residues in wild fish from cotton-growing areas of New South Wales, Australia. J. Environ. Qual. 30: 740-750.

Kumari, B., V. K. Madan, R. Kumar, and T. S. Kathpal. 2002. Monitoring of seasonal vegetables for pesticide residues. Environ. Monit. Assess. 74: 263-270.

LeOra Software. 1987. POLO-PC a user's guide to probit analysis or logit analysis. LeOra Software, Berkeley, CA.

Macleod Jr., W. D., and N. M. Buigues. 1964. Sesquiterpenes. 1. Nootkatone, a new grapefruit flavor constituent. J. Food Sci. 29: 565-568.

Maistrello, L., G. Henderson, and R. A. Laine. 2001a. Efficacy of vetiver oil and nootkatone as soil barriers against Formosan subterranean termite (Isoptera: Rhinotermitidae). J. Econ. Entomol. 94: 1532-1537.

Maistrello, L., G. Henderson, and R. A. Laine. 2001b. Effects of nootkatone and a borate compound on Formosan subterranean termite (Isoptera: Rhinotermitidae) and its symbiont protozoa. J. Entomol. Sci. 36: 229-236.

Maistrello, L., G. Henderson, and R. A. Laine. 2002. Comparative effects of vetiver oil, nootkatone and disodium octaborate tetrahydrate on Coptotermes formosanus and its symbiotic fauna. Pest. Manage. Sci. 59: 58-68.

Miyazawa, M., H. Tougo, and M. Ishihara. 2001. Inhibition of acetylcholinesterase activity by essential oil from Citrus paradisi. Nat. Prod. Lett. 15: 205-210.

Nix, K. E., G. Henderson, and R. A. Laine. 2003. Field evaluation of nootkatone and tetrahydronootkatone as wood treatment against Coptotermes formosanus. Sociobiology 42: 413-424.

Osbrink, W.L.A., A. R. Lax, and R. J. Brenner. 2001. Insecticide susceptibility in Coptotermes formosanus and Reticulitermes virginicus (Isoptera: Rhinotermitidae). J Econ. Entomol. 94: 1217-1228.

Pickett, J. A., L. J. Wadhams, M.-H. Pham-Delègue, and C. M. Woodcock. 2001. New opportunities for commercial exploitation of insect olfactory reception. In The 18th Annual Meeting of the International Society of Chemical Ecology, Granlibakken Resort, Lake Tahoe, CA, 2001 July $7-12$.

SAS Institute 1989. SAS/STAT user's guide. SAS Institute, Cary, NC.

Shaw, P. E., and C. W. Wilson, 3rd. 1981. Importance of nootkatone to the aroma of grapefruit oil and the flavor of grapefruit juice. J. Agric. Food Chem. 29: 677-679.

Sim, M., A. Forbes, J. McNeil, and G. Roberts. 1998. Termite control and other determinants of high body burdens of cyclodiene insecticides. Arch. Environ. Health 53: 114121.

Smith, V. K. 1979. Improved techniques designed for screening candidate termiticides on soil in the laboratory. J. Econ. Entomol. 72: 877-879.

Spink, W. T. 1967. The Formosan subterranean termite in Louisiana. Circ No. 89, Louisiana. Agric. Exp. Stn., Baton Rouge, LA.

Su, N.-Y., V. Chew, G. S. Wheeler, and R. H. Scheffrahn. 1997. Comparison of tunneling responses into insecticide-treated soil by field populations and laboratory 
groups of subterranean termites (Isoptera: Rhinotermitidae). J. Econ. Entomol. 90: 503-509.

Su, N.-Y., and J. P. La Fage. 1986. Effects of starvation on survival and maintenance of soldier proportion in laboratory groups of the Formosan subterranean termite, Coptotermes formosanus (Isoptera: Rhinotermitidae). Ann. Entomol. Soc. Am. 97: 312-316.

Su, N.-Y., and M. Tamashiro. 1987. An overview of the Formosan termite (Isoptera: Rhinotermitidae) in the world, pp. 3-15. In M. Tamashiro, and N.-Y. Su [eds.], Biology and control of the Formosan subterranean termite. Res. Ext. Series 083. College of Tropical Agriculture and $\mathrm{Hu}-$ man Resources, University of Hawaii, Honolulu, HI.

Sun, J., J. R. Fuxa, and G. Henderson. 2003. Virulence and in vitro characteristics of pathogenic fungi isolated from soil by baiting with Coptotermes formosanus (Isoptera: Rhinotermitidae). J. Entomol. Sci. 38: 342-358.

Supriana, N. 1983. Preliminary tests on the effect of naturally occurring chemicals on termites. Document IRG/ WP/1181. The International Research Group on Wood Preservation, Queensland, Australia.

Suszkiw, J. 1998. The Formosan termite: a formidable foe. Agric. Res. 46: 4-9.
Tamashiro, M., J. R. Yates III, and R. H. Ebesu. 1987. The Formosan subterranean termite in Hawaii: problems and control, pp. 15-22. In M. Tamashiro and N.-Y. Su [eds.], Biology and control of the Formosan subterranean termite. Res. Ext. Series 083. College of Tropical Agriculture and Human Resources, University of Hawaii, Honolulu, HI.

Tassaneeyakul, W., L.-Q. Guo, K. Fukuda, T. Ohta, and Y. Yamazoe. 2000. Inhibition selectivity of grapefruit juice components on human cytochromes P450. Arch. Biochem. Biophys. 378: 356-363.

Wilkins, C. K. 1992. Naturally occurring anti-termite compounds. Mater. Org. 27: 47-66.

Zhu, B.C.R., G. Henderson, F. Chen, H. Fei, and R. A. Laine. 2001a. Evaluation of vetiver oil and seven insect-active essential oils against Formosan subterranean termites. J. Chem. Ecol. 27: 1617-1625.

Zhu, B.C.R., G. Henderson, F. Chen, L. Maistrello, and R. A. Laine. 2001b. Nootkatone is a repellent for Formosan subterranean termite (Coptotermes formosanus). J. Chem. Ecol. 27: 523-531. Recei.
Received for publication 7 March 2003; accepted 8 October 